\title{
Was the unusual solar cycle at the end of the XVIII century a result of phase asynchronization?
}

\author{
N. V. Zolotova and D. I. Ponyavin
}

\author{
Institute of Physics, Saint-Petersburg State University, Russia \\ e-mail: [ned;ponyavin] @geo.phys.spbu.ru
}

Received 19 April 2007 / Accepted 22 May 2007

\section{ABSTRACT}

\begin{abstract}
Aims. The problem of the unusual sunspot cycle in 1784-1799 is considered. Why was the length of the 4th solar cycle exceptionally large? Was it really composed of two shorter cycles? Resolving this puzzle seems to be very important for modern dynamo theories trying to explain the solar cyclicity.

Methods. We propose a possible scenario for the solar activity behavior during the 4th solar cycle. Analogies with the phase asynchronization observed at the time of the 20th solar cycle are presented.

Results. We suggest that the length of the 4th solar cycle can be explained by outstanding phase asynchrony between Northern and Southern hemispheric activities reaching a delay up to 4.5 years. The main effect of such asynchronization results in strong North-South asymmetry during the course of the ascending phase of the solar cycle 4.
\end{abstract}

Key words. Sun: activity - Sun: sunspots - Sun: magnetic fields

\section{Introduction}

The uncertainty and scarcity of historical records define the main difficulties in reconstructing any solar activity in the past. Even a small inaccuracy in sunspot observations can dramatically change estimations, phase dynamics, and other solar cycle properties because of the lack of statistics. Therefore careful routine data mining is an essential part of solar reconstruction.

Not long ago, a sensational suggestion was made by Usoskin et al. (2001) concerning the loss of one solar cycle at the beginning of the Dalton minimum. It was supposed that the generally accepted 4th solar cycle, according to Zürich numeration, is actually a superposition of two cycles. The traditional, exceptionally long 4th solar cycle breaks the famous Gnevyshev-Ohl rule and leads to the phase catastrophe (Vitinsky et al. 1986; Kremliovsky 1994; Usoskin \& Mursula 2003). Two short cycles (9 and 7 years in lengths) were suggested instead of this kind of abnormally long cycle.

Krivova et al. (2002) have checked this hypothesis by performing some statistical tests of available sunspot records and also considered ${ }^{10} \mathrm{Be},{ }^{14} \mathrm{C}$, and auroral proxy data. According to their results, the presumably lost cycle appears even more peculiar than the standard 4th cycle. In reply Usoskin et al. (2003) re analyzed of sunspot statistics. They show that the average level of sunspot activity in 1792-1793 was similar to what it was around sunspot cycle minima during the more recent, wellobserved years 1850-1996, but it is significantly different from the activity either in the mid-declining phase or around sunspot maxima.

The other implication of the new suggested cycle was the Waldmeier rule relating the amplitude of a solar cycle to the length of its phases. Usoskin et al. (2002) show that the new cycle does not change the cycle-length distribution and only slightly modifies the formal anti-correlation in the Waldmeier effect.
At the same time, Veselovsky \& Tarsina (2002) have presented the approximate empirical formula that relates the duration of the ascending phase and the maximal sunspot numbers for a given solar cycle. Yakovchouk \& Veselovsky (2006) extend this analysis for the group sunspot numbers and sunspot area datasets. Concerning the lost-cycle hypothesis they show that including a new short cycle violates Waldmeier relationships. They go on demonstrate that since 1500 there has been no cycle with duration of ascending phase shorter than two years. But introducing a new cycle reduces the duration of the ascending phase to 1.9 years. Analyzing the auroral data prepared by Silverman (1983) for the period from 1720 to 1850 , Yakovchouk \& Veselovsky (2006) trace auroral peaks attributed to descending solar cycle phases. These peaks seemingly reveal a complex geomagnetic response to solar activity output, but do not mark the new sunspot cycle.

According to the modern view, geomagnetic activity consists of two parts: one associated with transient geoeffective phenomena, which follows the solar activity cycle, and a second associated with quasistationary, recurrent solar wind streams, which occurred after the solar activity maximum (e.g. Simon \& Legrand 1986; Ruzmaikin \& Feynman 2001; Hathaway \& Wilson 2006).

The problem of 4 th cycle still remains unsolved. Arguments for and against the lost-cycle hypothesis hitherto give rise to doubtful statistics and contradictory results for the Waldmeier effect. Our paper is an attempt to explain the nature of the exceptionally long 4th solar cycle as a result of the outstanding phase asynchrony of the north-south solar activity in 1784-1799.

\section{Sunspot asynchronization in the 4th and 20th cycles}

In the original paper, Usoskin et al. (2001) analyzed the group sunspot numbers (GSN) provided by Hoyt \& Schatten (1998), 


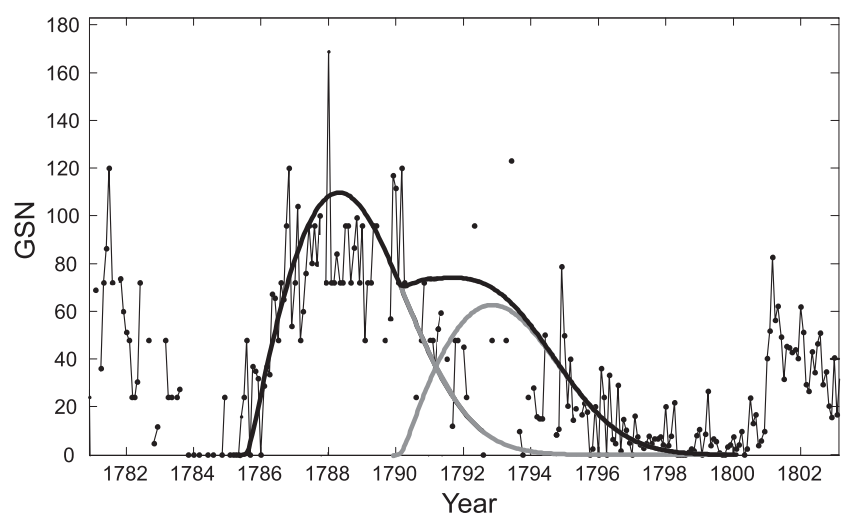

Fig. 1. Sunspot activity circa the solar cycle 4 . Thin black curve corresponds to the monthly group sunspot numbers. Suggested smoothed sunspot activities for hemispheres are shown by gray thick curves, and their sum - by the black thick curve.

due to their higher homogeneity and accuracy against sunspot numbers. In this work we also used the GSN data tabulated by the National Geophysical Data Center.

We suppose that, at the time of 1785-1788, the sunspot cycle was first started in one hemisphere. Active regions in the other hemisphere began to emerge only few years later.

Figure 1 presents the model of sunspot asynchrony during the course of the 4 th cycle. It indicates both the monthly group sunspot numbers and the suggested smoothed sunspot activities for hemispheres, along with their sum.

Shapes of the hemispheric cycles can be described in terms of a simple function with four parameters (Hathaway et al. 1994):

$f(t)=\frac{a\left(t-t_{\mathrm{o}}\right)^{3}}{\exp \left(\frac{\left(t-t_{\mathrm{o}}\right)^{2}}{b^{2}}\right)-c}$,

where parameter $a$ represents the amplitude, $b$ is related to the time in years from the minimum to the maximum of a cycle, $c$ gives the asymmetry of the cycle, and $t_{\mathrm{o}}$ denotes the starting time.

For the leading hemisphere we take $a=7, b=3, c=0.99$, and $t_{\mathrm{o}}=1785.5$. For the driven hemisphere $-a=4, b=3$, $c=0.99$, and $t_{\mathrm{o}}=1790$. Here the time delay between the onsets of hemispheric cycles is 4.5 years. The lengths of the cycles are about 9 years. To better fit available data, we suppose that sunspot hemispheric activities were different in amplitude. This suggestion comes from analyzing the monthly GSN that were overestimated and uncertain in 1792-1793.5 (Usoskin et al. 2001).

Thus we suggest that the solar cycle 4 is composed of two relatively short hemispheric cycles time-shifted each other by a few years. Its length depends on phase synchronization between opposite hemispheres. Due to phase asynchrony, an exceptionally long 4th cycle disrupted phase dynamics and caused phase catastrophe.

Another case of strong phase asynchrony was observed during the 20th cycle (Fig. 2a). Curious similarities between sunspot patterns appear to be repeated every 16 solar cycles (Fairbridge $\&$ Hameed 1983). Here we have used smoothed (by moving oneyear averages), monthly means of sunspot area for the northern and southern hemispheres. The length of the 20th cycle over the total smoothed sunspot area is 12.5 years. It is the longest cycle since 1874 . Also the cycle has a long descending phase of about 6.5 years. Southern hemispheric activity produced peaks in 1972
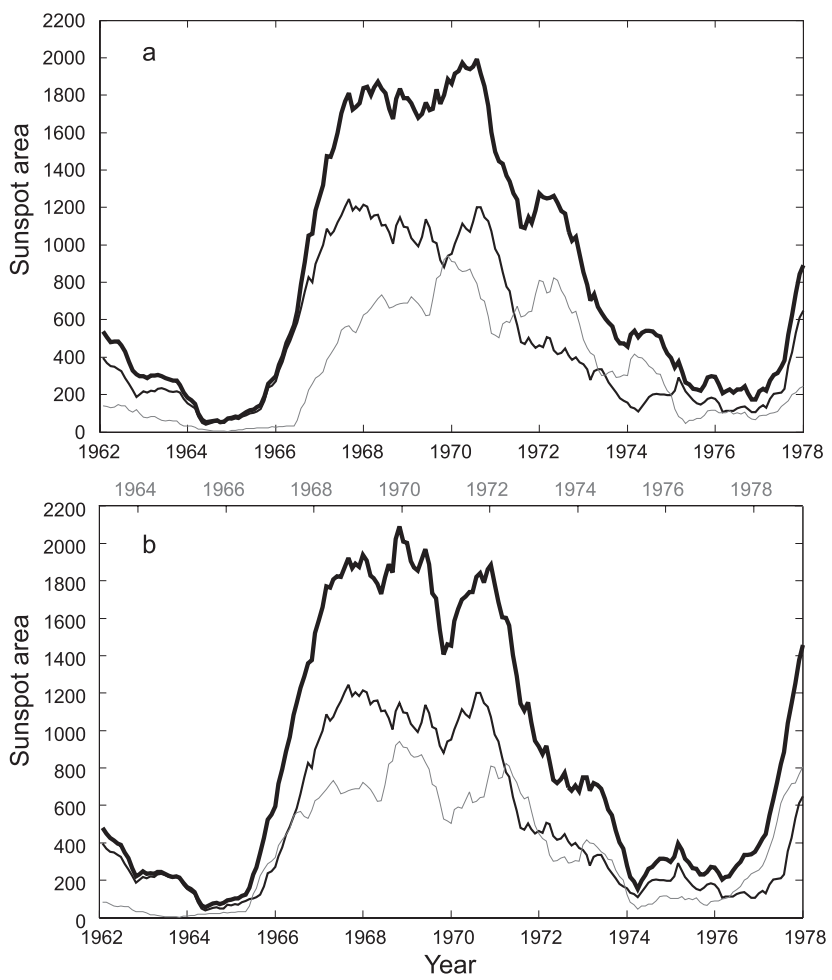

Fig. 2. Sunspot activity circa the solar cycle 20. a) Black thin curve is smoothed monthly means of a sunspot area for the northern and gray thin curve - for the southern hemisphere. The total sunspot area is shown by a thick black curve. b) The same, but with a time-shift of the southern hemispheric activity.

and 1974.5 that are similar to the descending phase of the 4th cycle, where we suspect significant phase asynchronization.

Lag in the onsets of sunspot activities between the northern and southern hemispheres was more than a year in the beginning of the 20th cycle. Zolotova \& Ponyavin (2006) have shown that sunspot phase asynchronization between the cycles 19-20 was the highest since 1874 (up to 3 years).

Figure $2 \mathrm{~b}$ represents results of fitting hemispheric solar cycle shapes according to activity onsets. The South was shifted backward to 13 months and the time axis for the South is marked in the top panel. It is seen that some small delay significantly changed the common shape of the solar cycle 20 forming the more pronounced double-peak structure and shorter descending phase. It seems that the duration of the cycle due to their phase shift between opposite hemispheres is reduced to 10 years.

\section{Length of solar cycle. Discussion}

Here we discuss how the significant effect of sunspot hemispheric asynchronization is. How strong the duration of solar cycle depends on the phase relationships between hemispheric activities? Figure 3a demonstrates the variability of cycle durations with time. It shows the lengths of group sunspot cycles defined as the time difference between two successive sunspot minima (Usoskin \& Mursula 2003) and the lengths of smoothed monthly sunspot area cycles defined using sunspot minima. It is interesting to note that the two biggest deviations in the sunspot synchronization between hemispheres occur during the solar cycles 14-15 and 19-20 (Zolotova \& Ponyavin 2006). Figure 3b illustrates the lengths of smoothed sunspot area cycles defined from minimum to minimum for both hemispheres and for the total Sun (see Table 1). Uncertainties in determining the cycle 
Table 1. Lengths of sunspot area cycles defined using minima for the hemispheres and the total solar surface.

\begin{tabular}{cccc}
\hline \hline $\begin{array}{c}\text { Number of } \\
\text { cycle }\end{array}$ & $\begin{array}{c}\text { Length of the total } \\
\text { sunspot cycle }\end{array}$ & $\begin{array}{c}\text { Length of the Northern } \\
\text { sunspot cycle }\end{array}$ & $\begin{array}{c}\text { Length of the Southern } \\
\text { sunspot cycle }\end{array}$ \\
\hline 12 & 11.17 & 10.42 & 11.58 \\
13 & 11.16 & 11.42 & 11.08 \\
14 & 12.34 & 11.59 & 12.17 \\
15 & 9.91 & 11.16 & 10.08 \\
16 & 10.17 & 10.25 & 9.58 \\
17 & 10.58 & 10.5 & 10.83 \\
18 & 10 & 9.92 & 10.33 \\
19 & 10.09 & 10.17 & 10.59 \\
20 & 12.5 & 12.41 & 10.41 \\
21 & 9.83 & 9.17 & 11.37 \\
22 & 9.58 & 10.58 & 9.25 \\
\hline
\end{tabular}
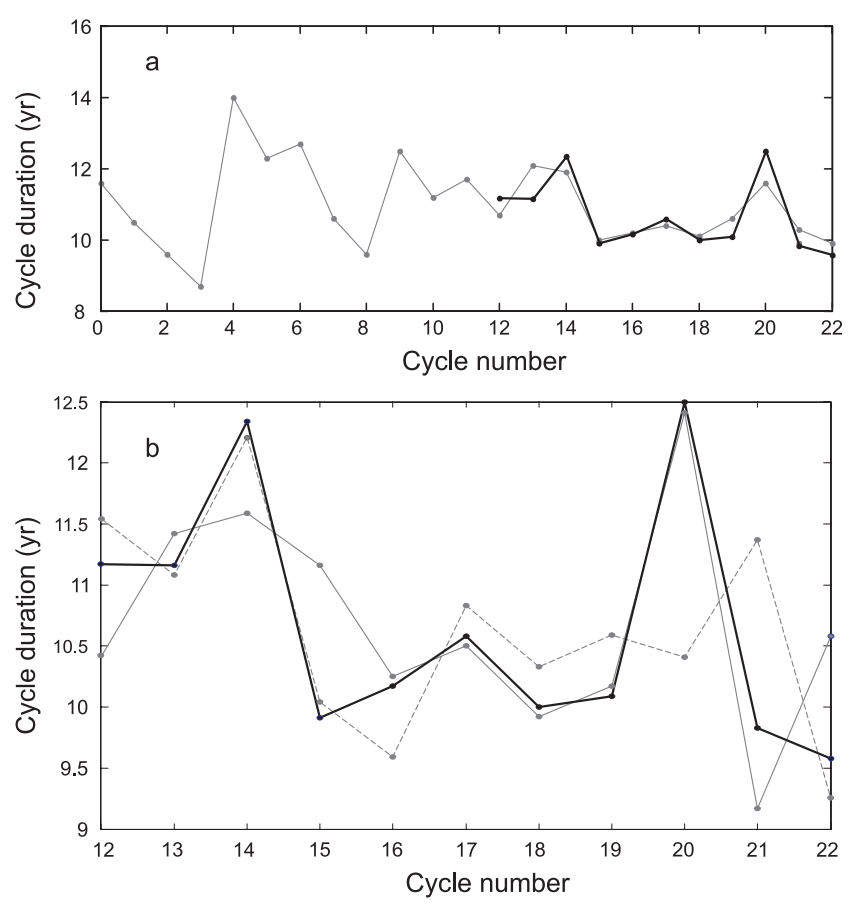

Fig. 3. a) Durations of solar cycles versus time. The gray thin curve corresponds to the lengths of group sunspot cycles. The black thick curve shows lengths of smoothed sunspot area cycles. b) The lengths of smoothed monthly sunspot area cycles. The gray solid curve corresponds to the northern hemisphere and gray dashed curve - to the southern hemisphere. Thick black curve represents the total Sun (see Table 1).

length from minimum to minimum extend up to more than a year (Mursula \& Ulich 1998).

It is seen that the duration of the total cycle 14 was defined by the length of the southern hemispheric cycle. During the solar cycle 20, the opposite was formed by the northern hemisphere. Thus, in spite of large phase asynchrony observed at the beginning of the 20th cycle, its length strongly depends on the duration of the northern cycle.

According to Fig. $3 \mathrm{~b}$ the length of the total sunspot cycle $(\mathrm{N}+\mathrm{S})$ can be less than the lengths for the northern and southern hemispheres as happened in cycles 15 and 19 (see Table 1). This illusory contradiction comes from fluctuations of solar activity and phase relationships between time series around the sunspot minimum.

As an example, Fig. 4a presents the time profile of the solar cycle 19, and the color labelling is the same as in Fig. 2.
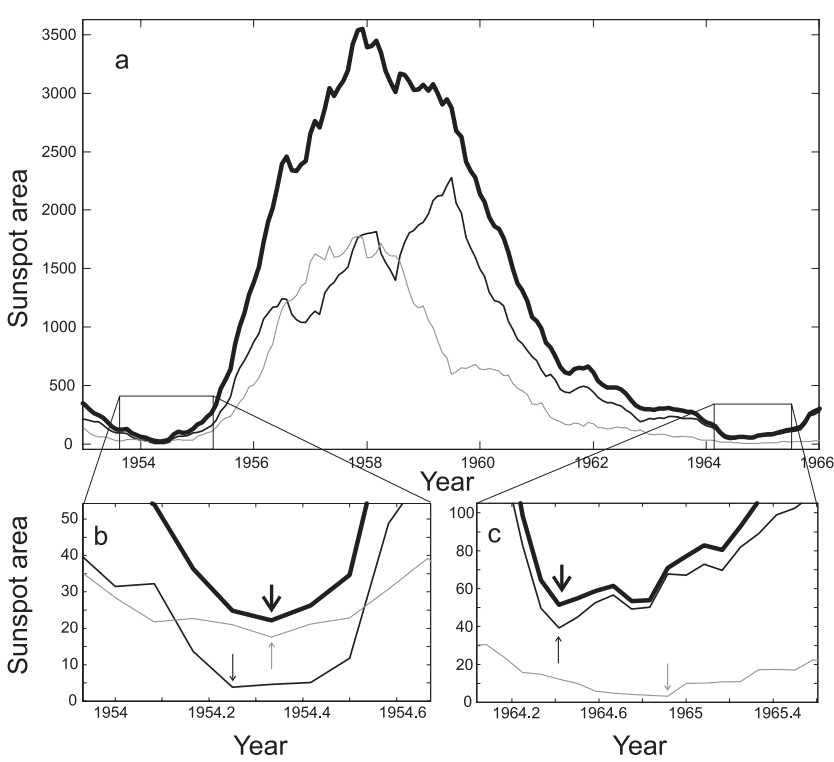

Fig. 4. Sunspot activity circa the solar cycle 19. a) The smoothed monthly means of sunspot area for the northern (black thin) and southern hemispheres (gray thin). The total sunspot area is shown by a thick black curve. b) Segment of the sunspot activity minimum at the beginning of the solar cycle 19. Arrows mark time moments in which hemispheric and total activities have the lowest values. c) The same but for the end of the 19th cycle.

Figures $4 \mathrm{~b}$ and $4 \mathrm{c}$ show two zoomed successive minima. Arrows mark those time moments when hemispheric and total sunspot activities have the lowest values. The difference between these dates corresponds to the cycle length. It is seen that the activity minimum of the northern hemisphere (Fig. 4b) leads activity minima of the southern hemisphere and total Sun to 0.08 year. At the end of the 19th cycle, in contrast the south minimum delays north and total activity minima to 0.5 year (Fig. 4c). As a result the length of the total 19th cycle is 10.09 ; for the northern hemisphere it is 10.17 and for the southern hemisphere 10.59 .

The idea that strong asynchrony between northern and southern activities existed during the course of solar cycle 4 can be checked by analyzing the historic data. The asynchrony should result in a strong North-South asymmetry during the first years of solar cycle 4 . We hope that later findings (e.g. Vaquero et al. 2007) will prove (or disprove) our hypothesis.

Noteworthy is the outstanding discovery by Ribes \& Nesme-Ribes (1993) of strong North-South asymmetry during the Maunder Minimum (1645-1715) that stimulated dynamo 
theories trying to explain solar secular variations (e.g. Sokoloff \& Nesme-Ribes 1994; Serre \& Nesme-Ribes 2000; Wang \& Sheeley 2003; Charbonneau 2005).

\section{Conclusions}

In this paper we have proposed a scenario to explain the origin of the exceptionally long 4th solar cycle. We suggest that in 1784-1799 no cycle was lost. The duration of the 4th cycle can be the result of the outstanding phase asynchrony of the north-south solar activity. We suppose that the main contribution of asynchrony was significant the difference in the onset times of solar cycles in the northern and southern hemispheres. The difference between time onsets reached at least 3 years. The sunspot maximum of the leading hemisphere was between 1788 and 1790, and for driven hemisphere between 1793 and 1795 . The descending phase of the solar cycle after 1795 can be mainly formed either by activity presented only in one hemisphere or by activities in both hemispheres as happened in the 20th cycle. Phase asynchronization and delay disappeared during the descending phase.

We suggest that suppressed and delayed activity in the driven hemisphere at the end of XVIII century can be one reason for the solar cycle length increasing and subsequent grand minima of the solar activity. Some evidence of the length increasing just before the prolonged minimum, like Dalton and Maunder, was demonstrated early by Chernosky (1955) and Frick et al. (1997).

A similar situation for the big phase delay was observed during the ascending phase of solar cycle 20. However, the length of this cycle is defined mainly by the length of the cycle in the northern hemisphere. Another cycle whose length was defined by predominant hemispheric activity is the 14th cycle.

Finally we would like to note that coupling between lengths of the cycles and phase hemispheric asynchrony are significant but most likely have a nonlinear nature. To determine the solar cycle length not only the cycle overlapping but phase hemispheric relationships also have to be taken into account.

Acknowledgements. We thank the anonymous referee for his/her useful comments that helped to improve the paper. This research was supported by an INTAS Fellowship Grant for Young Scientists Ref. No. 06-1000014-6022.

\section{References}

Charbonneau, P. 2005, Sol. Phys., 229, 345

Chernosky, E. J. 1955, PASP, 67, 117

Fairbridge, R. W., \& Hameed, S. 1983, AJ, 88, 867

Frick, P., Galyagin, D., Hoyt, D. V., et al. 1997, A\&A, 328, 670

Hathaway, D. H., \& Wilson, R. M. 2006, Geophys. Res. Lett., 33, 18101

Hathaway, D. H., Wilson, R. M., \& Reichmann, E. J. 1994, Sol. Phys., 151, 177

Hoyt, D. V., \& Schatten, K. 1998, Sol. Phys., 179, 189

Kremliovsky, M. N. 1994, Sol. Phys., 151, 351

Krivova, N. A., Solanki, S. K., \& Beer, J. 2002, A\&A, 396, 235

Mursula, K., \& Ulich, Th. 1998, Geophys. Res. Lett., 25, 1837

Ribes, J. C., \& Nesme-Ribes, E. 1993, A\&A, 276, 549

Ruzmaikin, A., \& Feynman, J. 2001, J. Geophys. Res., 106, 15783

Serre, T., \& Nesme-Ribes, E. 2000, A\&A, 360, 319

Silverman, S. M. 1983, J. Geophys. Res., 88, 8123

Simon, P. A., \& Legrand, J.-P. 1986, A\&A, 155, 227

Sokoloff, D., \& Nesme-Ribes, E. 1994, A\&A, 288, 293

Usoskin, I. G., \& Mursula, K. 2003, Sol. Phys., 218, 319

Usoskin, I. G., Mursula, K., \& Kovaltsov, G. A. 2001, A\&A, 370, L31

Usoskin, I. G., Mursula, K., \& Kovaltsov, G. A. 2002, Geophys. Res. Lett., 29, 36

Usoskin, I. G., Mursula, K., \& Kovaltsov, G. A. 2003, A\&A, 403, 743

Vaquero, J. M., Trigo, R. M., Gallego, M. C., \& Moreno-Corral, M. A. 2007, Sol. Phys., 240, 165

Veselovsky, I. S., \& Tarsina, M. V. 2002, Adv. Space Res., 29, 417

Vitinsky, Yu. I., Kopecky, M., \& Kuklin, G. V. 1986, Statistics of Sunspot Activity (Moscow: Nauka)

Wang, Y.-M., \& Sheeley, N. R., Jr. 2003, ApJ, 591, 1248

Yakovchouk, O. S., \& Veselovsky, I. S. 2006, in Quasiperiodical processes on the Sun and their geoeffective manifestations, ed. A. V. Stepanov, A. A. Soloviev, \& Yu. A. Nagovitsyn (St. Petersburg), Proc. X Pulkovo Int. Conf. Solar Phys., 155

Zolotova, N. V., \& Ponyavin, D. I. 2006, A\&A, 449, L1 